\title{
ARTICLE
}

Received 7 Jul 2014 | Accepted 18 Sep 2014 | Published 31 Oct $2014 \quad$ DOI: 10.1038/ncomms6335

\section{Biologically inspired pteridine redox centres for rechargeable batteries}

Jihyun Hong ${ }^{1, \star}$, Minah Lee ${ }^{2, \star}$, Byungju Lee ${ }^{1}$, Dong-Hwa Seo ${ }^{1, \dagger}$, Chan Beum Park $^{2}$ \& Kisuk Kang ${ }^{1,3}$

The use of biologically occurring redox centres holds a great potential in designing sustainable energy storage systems. Yet, to become practically feasible, it is critical to explore optimization strategies of biological redox compounds, along with in-depth studies regarding their underlying energy storage mechanisms. Here we report a molecular simplification strategy to tailor the redox unit of pteridine derivatives, which are essential components of ubiquitous electron transfer proteins in nature. We first apply pteridine systems of alloxazinic structure in lithium/sodium rechargeable batteries and unveil their reversible tautomerism during energy storage. Through the molecular tailoring, the pteridine electrodes can show outstanding performance, delivering $533 \mathrm{Wh} \mathrm{kg}^{-1}$ within $1 \mathrm{~h}$ and $348 \mathrm{Wh} \mathrm{kg}^{-1}$ within $1 \mathrm{~min}$, as well as high cyclability retaining $96 \%$ of the initial capacity after 500 cycles at $10 \mathrm{Ag}^{-1}$. Our strategy combined with experimental and theoretical studies suggests guidance for the rational design of organic redox centres.

\footnotetext{
${ }^{1}$ Department of Materials Science and Engineering, Research Institute of Advanced Materials (RIAM), Seoul National University, 1 Gwanak Road, Seoul 151-742, Republic of Korea. ${ }^{2}$ Department of Materials Science and Engineering, Korea Advanced Institute of Science and Technology (KAIST), 335 Science Road, Daejeon 305-701, Republic of Korea. ${ }^{3}$ Center for Nanoparticle Research, Institute for Basic Science (IBS), Seoul National University, 1 Gwanak Road, Seoul 151-742, Republic of Korea. * These authors contributed equally to this work. $\dagger$ Present address: Department of Materials Science and Engineering, Massachusetts Institute of Technology, Cambridge, Massachusetts 02139, USA. Correspondence and requests for materials should be addressed to C.B.P. (email: parkcb@kaist.ac.kr) or to K.K. (email: matlgen1@snu.ac.kr).
} 
O rganic materials represent an attractive alternative to transition metal oxide cathodes in conventional rechargeable batteries because of their light weight, structural flexibility and chemical diversity ${ }^{1,2}$. In particular, organic molecules that mimic redox centres in biological energy transduction have gained a great deal of attention for their sustainability and universal availability in natural systems $s^{3,4}$. For instance, carbonyl-based compounds are the most well-known examples of bio-inspired redox centres, imitating quinone cofactors in nature, such as plastoquinone and ubiquinone, which are involved in the electron transport chain of chloroplasts and mitochondria ${ }^{5-9}$. Previous studies on these compounds have shown promising electrochemical properties with high energy densities ${ }^{10-13}$. Recent efforts have enabled the carbonyl-based organic electrodes to have high power as well as stable cycling performance, hinting at a practical feasibility for a bio-inspired design of energy storage materials ${ }^{10,14-17}$.

As a wide range of ion-coupled electron transfer reactions exists in biological energy conversion, the characterization of these redox systems may provide novel methods of electrochemical energy storage with versatile functionalities. In living organisms, heterocyclic molecules containing a pteridine $(1,3,5,8$ tetraazanaphthalene) nucleus composed of fused pyrimidine and pyrazine rings are widely occurring redox centres ${ }^{18,19}$. Among such molecules, flavin cofactors including FAD and flavin mononucleotide are one of the most vital compounds that catalyse biotransformation and energy transfer reactions ${ }^{20}$. During photosynthesis and cellular respiration, for example, flavin cofactors serve as a key redox centre facilitating protoncoupled electron transfer reactions for energy transduction. During the reaction, redox cycling occurs at the diazabutadiene of the isoalloxazine moiety ${ }^{21,22}$. On the basis of this electrochemical cellular metabolism, we recently proposed that natural flavin cofactors can be applied to lithium-storing electrodes in rechargeable batteries ${ }^{23}$. Although flavin-based electrodes can reversibly facilitate lithium-coupled electron transfer in batteries, their inadequate performance in terms of energy density and cycle stability requires further optimization along with in-depth studies regarding the underlying energy storage mechanism in the battery systems.

In this report, we present a molecular simplification strategy to design a minimal redox-active unit of the diazabutadiene redox cycle using chemical tailoring. The resulting pteridine derivatives in the alloxazinic structure are first used in lithium and sodium rechargeable battery systems as a novel class of organic cathodes. Spectroscopic and computational studies reveal differences in the underlying energy storage mechanisms between pristine isoalloxazic and derived alloxazinic systems. Furthermore, by introducing nanocarbons as a conductive scaffold for molecule immobilization during electrode preparation, we demonstrate that the pteridine derivatives exhibit outstanding electrochemical performance in terms of power and cyclability in battery operations.

\section{Results}

Molecular tailoring of pteridine systems for energy storage. To increase energy density, we simplified the chemical structure of flavin molecules by removing non-redox-active substituents from the molecules while maintaining the redox-active moiety (Fig. 1a). The centre of redox reactivity in flavins is the isoalloxazine ring system, specifically the conjugated diazabutadiene (N5-C4a-C10a-Ni1) region that facilitates the lithium-coupled electron transfer reaction via sequential reduction of N5 and N1 (Fig. 1b $)^{23}$. Without the ribityl substituents, which yields a simpler molecule lumichrome (7,8-dimethylalloxazine, LC), flavin could no longer maintain its isoalloxazinic form. Rather, LC formed an alloxazinic structure, in which N1 and N3 (instead of N10 and N3) are substituted as shown in Fig. 1a (ref. 24). Although the structures of isoalloxazinic and alloxazinic molecules are closely related, the chemical and physical properties of these two systems differ significantly ${ }^{25}$. For example, each system shows different photoemission bands: the excited-state lifetime of the isoalloxazinic structure is longer than that of the alloxazinic structure, and the isoalloxazinic structure exhibits stronger emission intensity. On the basis of these photophysical differences, previous studies on LC observed an interesting phenomenon called excited-state tautomerism, which is a structural isomerism from the alloxazinic to the isoalloxazinic type (Fig. 1c) ${ }^{26,27}$. However, few studies have explored the redox chemistry of LC. To the best of our knowledge, the reversible tautomeric reaction of LC during electrochemical reactions has not been demonstrated previously, which will be discussed below. We further searched for simpler forms of the pteridine redox centre by sequentially removing substituents, which are alloxazine (ALX, without the methyl groups at C7 and C8 of LC) and lumazine (LMZ, without the anellated benzo ring of ALX).

The redox properties of pteridine derivatives were first investigated with cyclic voltammetry using percholate electolytes in N,N-dimethylformamide (DMF) under $\mathrm{N}_{2}$ atmosphere (Fig. 1d). In tetrabutylammonium perchlorate (TBAP), which contains $\mathrm{TBA}^{+}$as noninteracting cations, pteridine derivatives exhibited two reduction and oxidation peaks in the cyclic voltammetry curves. The use of $\mathrm{LiClO}_{4}$ as a supporting electrolyte resulted in more complicated redox behaviour with positive shifts of the average redox potentials, which is attributed to the possible existence of interacting $\mathrm{Li}^{+}$cation ${ }^{16,28}$.

Lithium storage mechanism of LC. As the first step to utilize pteridine derivatives in lithium storage, we examined the electrochemical activity of the LC electrode versus lithium counter electrode in a coin-type cell. According to our galvanostatic measurements, LC/Li cells could reversibly take up and release two lithium ions and electrons. The specific capacity of LC/Li cell was $\sim 169 \mathrm{mAh} \mathrm{g}^{-1}$, equivalent to $1.53 \mathrm{Li}$ atoms per molecule between 1.5 and $3.6 \mathrm{~V}$ at a current density of $20.0 \mathrm{mAg}^{-1}$ (Fig. 2a). When we apply high temperature $\left(60^{\circ} \mathrm{C}\right)$ to the battery operation of LC, its capacity reached to $181 \mathrm{mAhg}^{-1}$ (Supplementary Fig. 1). The theoretical capacity corresponding to two $\mathrm{Li}$ atoms in the LC electrode is $221 \mathrm{mAhg}^{-1}$. Two sets of distinctive cathodic and anodic peaks were observed with average potentials of 2.50 and $2.35 \mathrm{~V}$ based on differential capacity curves (Fig. 2a, inset). The result indicated that the electrode reaction of LC followed two consecutive lithium insertion reactions with a relatively stable intermediate phase, which resembles the redox chemistry of riboflavins in lithium cells ${ }^{23}$. We also performed a galvanostatic intermittent titration technique (GITT) measurement under an intermittent discharge/charge for $1 \mathrm{~h}$ at a low current rate of $10.0 \mathrm{mAg}^{-1}$ with relaxation periods maintained for $5 \mathrm{~h}$. The GITT result in Fig. $2 \mathrm{~b}$ shows that the open-circuit voltage (OCV) of LC was nearly unchanged at $2.50 \mathrm{~V}$ during the first reduction by lithiation, while the OCV continuously decreased during the second stage of the reduction. During the recharge period, the OCV continuously increased to $2.50 \mathrm{~V}$, and then remained flat until the majority of lithium ions were extracted from LC. The flat potential in the electrochemical profile suggests that lithium insertion followed a two-phase-based reaction in the region, which is known to occur in conventional olivine $\mathrm{LiFePO}_{4}$ electrodes.

To characterize the redox mechanism of LC, we performed ex situ experimental and computational studies of the electrode during battery operation. Changes in the crystal structure of 
a

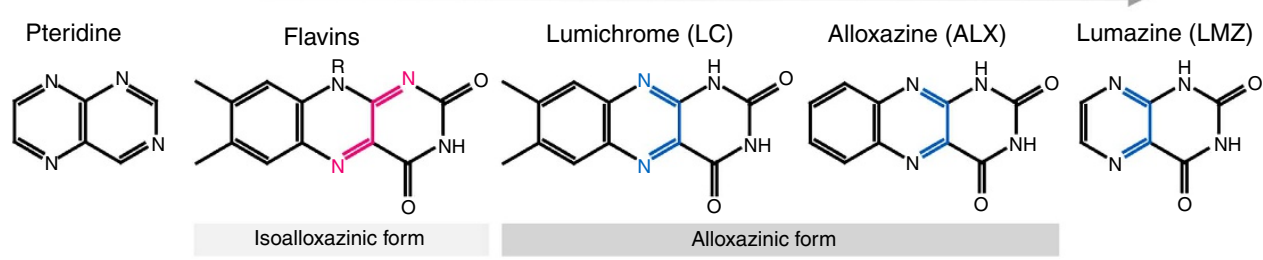

b

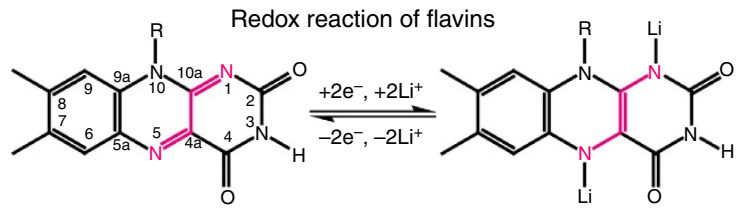

d

FAD : $\mathrm{R}=\mathrm{CH}_{2}(\mathrm{CHOH})_{3} \mathrm{CH}_{2} \mathrm{ADP}$ Riboflavin : $\mathrm{R}=\mathrm{CH}_{2}(\mathrm{CHOH})_{3} \mathrm{CH}_{2} \mathrm{OH}$

C<smiles></smiles>

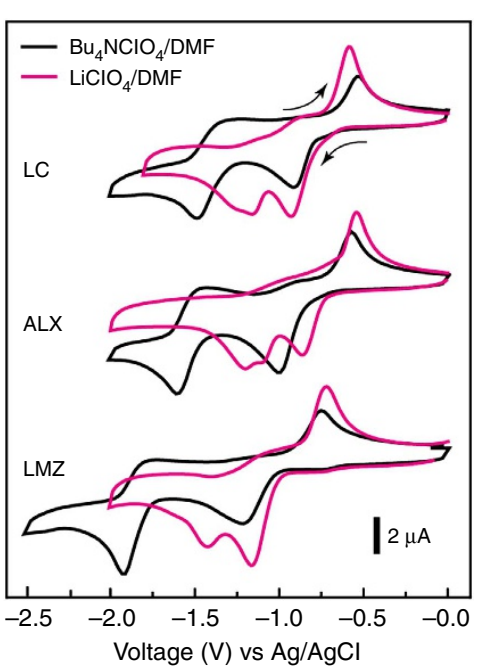

Figure 1 | Biologically inspired pteridine redox cofactors. (a) Molecular structures of pteridine derivatives as bioinpsired redox compounds for energy storage. (b) Redox mechanism of flavins inspired by biological energy transduction. (c) Reversible tautomerism of LC, which is a structural isomerism from the alloxazinc to the isoalloxazinic type. (d) Cyclic voltammograms of pteridine derivatives measured in DMF solutions containing TBAP (black) and $\mathrm{LiClO}_{4}$ (red) as electrolytes.

LC were first investigated by comparing X-ray diffraction patterns of LC electrodes at various states, including as-prepared, fully discharged and fully recharged states (Supplementary Fig. 2). We found that the as-prepared electrode comprising crystalline LC powders lost the long-range crystalline order on the first discharge and partially restored its original crystallinity on the recharge. The amorphization of the electrodes on cation insertion is often observed for the organic active materials, which is attributable to the relatively weak interactions (van der Waals force, hydrogen bond) among the molecules comprising the crystalline structure ${ }^{29,30}$. Nevertheless, the electrodes still retained the redox activity for continuous battery operation, which implies that the electrochemical activities are not dominated by the long-range crystalline order of organic electrode materials.

To identify the evolution of molecular structure during the electrochemical reactions, we performed various spectroscopic analyses. High-resolution X-ray photoelectron spectroscopy (XPS) scans of the electrodes confirmed the reversible lithium insertion and extraction in LC during battery cycling (Fig. 2c). In the Li 1s spectra, the lithium peaks reversibly evolved with the discharge and disappeared with subsequent charging of the LC electrode. The electron density in nitrogen and oxygen atoms of LC changed according to lithiation and delithiation, suggesting that these atoms are redox-active centres. The peak in the $\mathrm{N} 1 \mathrm{~s}$ spectra of the as-prepared electrode (full-width at half-maximum, FWHM: $2.23 \mathrm{eV}$ ) was broadened during the discharged state (FWHM: $2.57 \mathrm{eV}$ ) and then recovered after recharge (FWHM: $2.25 \mathrm{eV}$ ), which shifted the peak to lower energies and then back to the initial value. For the as-prepared electrode, the $\mathrm{N} 1 \mathrm{~s}$ spectrum could be deconvoluted into two peaks with the same FWHM of $1.60 \mathrm{eV}$ centred at 399.15 and $400.15 \mathrm{eV}$, which were assigned to conjugated $\left(\mathrm{sp}^{2}\right)-\mathrm{N}=$ and non-conjugated $\left(\mathrm{sp}^{3}\right)$
-NH- groups, respectively ${ }^{31}$. After discharge, an additional peak evolved at a lower binding energy, $398.40 \mathrm{eV}$, while the $-\mathrm{N}=$ peak $(399.15 \mathrm{eV})$ decreased, implying that reduction occurred at nitrogen atoms in the pyrazine ring. The new peak could be designated as $\mathrm{N}-\mathrm{Li}$, which was similar to that commonly observed in lithium azide ${ }^{32}$. For a recharged electrode, the reversible diminution of the $\mathrm{N}-\mathrm{Li}$ peak at $398.40 \mathrm{eV}$, as well as restoration of the $-\mathrm{N}=$ peak was observed, which is in agreement with the redox reaction of $\mathrm{LC}$ versus lithium. In the $\mathrm{O}$ 1s spectra, a slight downshift of the $\mathrm{O} 1 \mathrm{~s}$ binding-energy peak was detected in the discharged state, which indicated that the electron density of oxygen atoms also increased during lithiation-involved reduction of LC.

Ex situ Fourier transform infrared spectroscopy analysis shown in Fig. 2d further confirmed the reversible change in the molecular structure of LC during the redox reaction involving lithium insertion/extraction. As LC/Li cells were discharged and recharged, peaks at $1,278,1,561$ and $1,578 \mathrm{~cm}^{-1}$ corresponding to the vibrational modes of $\mathrm{C} 4 \mathrm{a}-\mathrm{C} 10 \mathrm{a}$ single bond and $\mathrm{C}=\mathrm{N}$ double bonds ${ }^{33}(v(\mathrm{~N} 10=\mathrm{C} 10 \mathrm{a}), v(\mathrm{~N} 5=\mathrm{C} 4 \mathrm{a}))$ gradually disappeared and reappeared. This result represents that the conjugated diazabutadiene motif $(-\mathrm{N}=\mathrm{C}-\mathrm{C}=\mathrm{N}-)$ participated in the redox reaction of LC, in agreement with the XPS results. In addition, we found that the vibrational modes of $\mathrm{C}=\mathrm{O}$ bonds ${ }^{34}$ were reversibly eliminated and restored not only for $\mathrm{C} 4$ but also for $\mathrm{C} 2$ at 1,697 and $1,723 \mathrm{~cm}^{-1}$, respectively, denoting that all lithium insertion reactions occur at the nitrogen atoms neighbouring oxygen atoms. This observation points to an interesting configurational change of the molecule during lithiation. Since N10 does not neighbor a C=O bond, as shown in Fig. 1c, it is expected that the N1 neighbouring $\mathrm{C}=\mathrm{O}$ participates in lithium accommodation, although the $\mathrm{N} 1$ atom is 
a

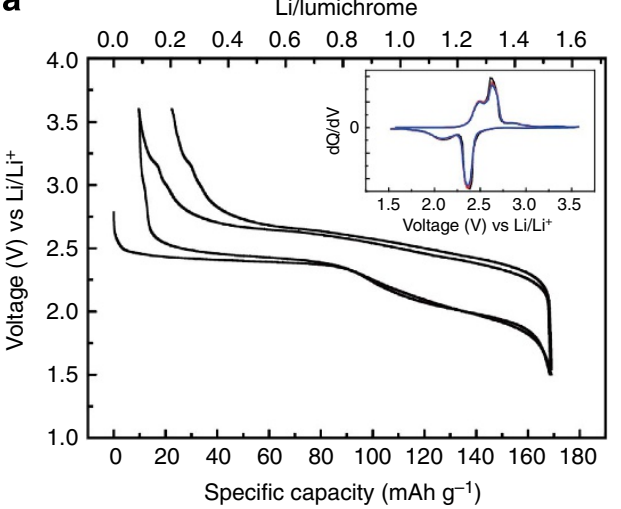

b

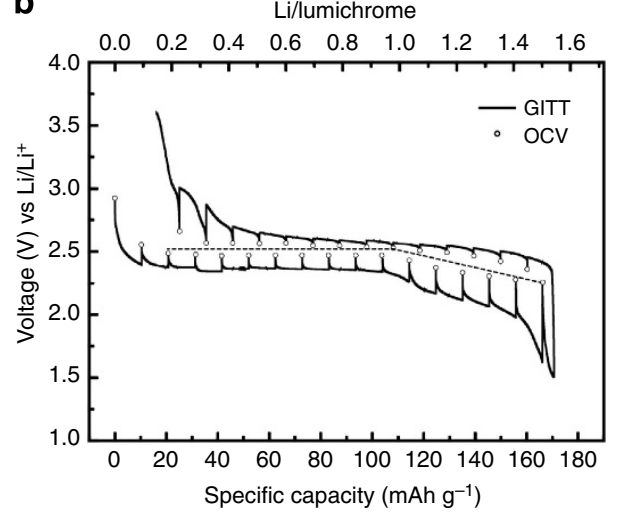

C
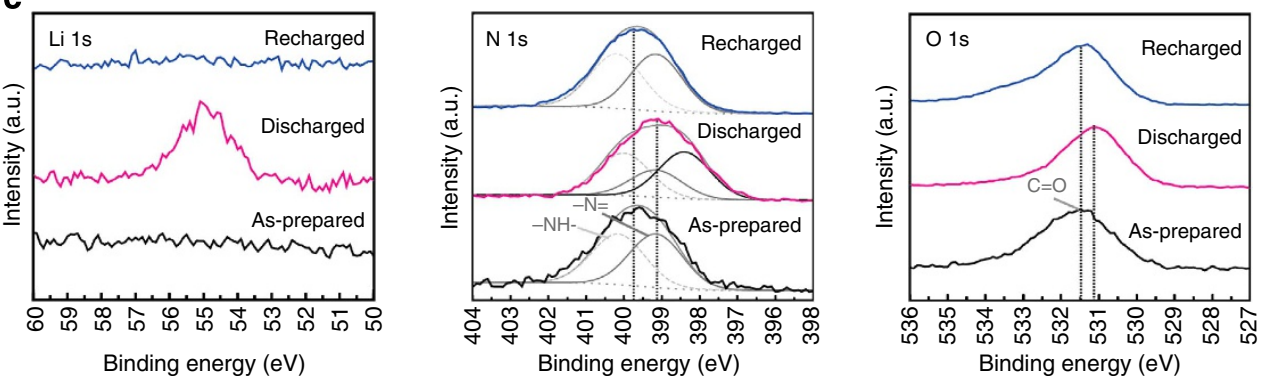

d
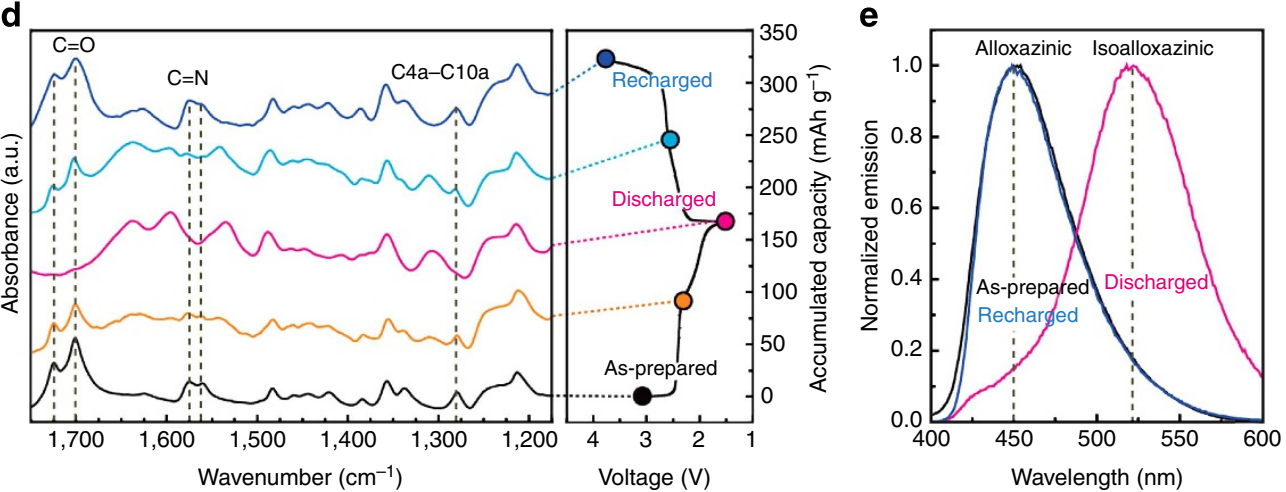

Figure 2 | Energy storage mechanism of lumichrome. (a) Capacity-voltage profiles and the corresponding differential capacity plots (inset) of LC/Li cells. (b) GITT profiles of the LC/Li cells. (c-e) Ex situ analyses of LC electrodes at different states of charge during battery cycling. (c) XPS local scan spectra of Li 1s, $\mathrm{N}$ 1s and $\mathrm{O}$ 1s regions, (d) FTIR spectra of $\mathrm{CN}$ and $\mathrm{CO}$ bonds and (e) emission spectra are reversibly switching on reduction and oxidation of the LC, which is indicative of the redox stability of alloxazinic systems.

already bound to a proton in the pristine alloxazinic form. This strongly suggests that tautomerism to the isoalloxazinic structure occurred electrochemically to form the $\mathrm{N} 1-\mathrm{Li}-\mathrm{O}-\mathrm{C} 2$ heterocyclic structure by accompanying proton transfer from N1 to N10.

We further monitored the configuration change of LC during reduction and oxidation in battery using fluorescence spectroscopy (Fig. 2e). After reduction (that is, discharge), the appearance of isoalloxazinic fluorescence at $520 \mathrm{~nm}$ and the disappearance of alloxazinic fluorescence at $450 \mathrm{~nm}$ at the discharged state were clearly observed, which agreed with previous reports on phototautomerism ${ }^{26,27}$. After recharging, the LC showed fluorescence at $450 \mathrm{~nm}$ again, suggesting that the tautomerism of the alloxazinic to the isoalloxazinic structure was completely reversible during the lithium-coupled redox reaction. The photo-induced tautomerism of LC has been well characterized $^{26,27}$, but the similar tautomerism to the isoalloxazinic structure that occured electrochemically during lithium-coupled electron reduction was unexpected.

Our experimental results on the redox mechanism of LC accompanying tautomerism were supported by density functional theory (DFT) calculations (B3LYP hybrid functional). First, we explored how many electrons could be maximally adopted and how they were distributed in LC by examining the highest occupied molecular orbitals (HOMO) of the molecule. The HOMO plot of a molecule provides qualitative information on its capability of accepting electrons in a given molecular structure ${ }^{35}$. When the LC molecule is reduced by accepting two electrons, effective electron delocalization occurs in the conjugated structure of reduced LC ( $\mathrm{LC}^{2-}$; Fig. 3a). Further reduction, however, does not allow the molecule to stabilize the electrons (Supplementary Fig. 3), indicating that LC is capable of a two-electron redox reaction. It was further noted that reduced $\mathrm{LC}\left(\mathrm{LC}^{2-}\right)$ was more stable in the isoalloxazinic form than in the pristine alloxazinic form by $0.299 \mathrm{eV}$.

To improve our understanding on the redox mechanism of LC, we compared DFT energies of possible lithium configurations for [LC] $\mathrm{Li}$ and $[\mathrm{LC}] \mathrm{Li}_{2}$ (Fig. 3b). It was found that [LC]Li containing $\mathrm{N} 5-\mathrm{Li}$ bond where the lithium ion also interacted with $\mathrm{O} 4$ was the most stable structure by more than $1.80 \mathrm{eV}$ among various possible configurations, and the alloxazinic structure was 
a

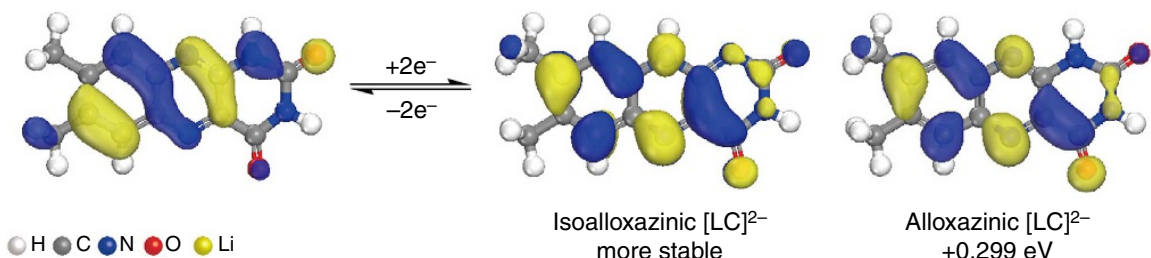

$O \mathrm{HOCON} \odot \mathrm{O} \odot \mathrm{Li}$ more stable $+0.299 \mathrm{eV}$

b
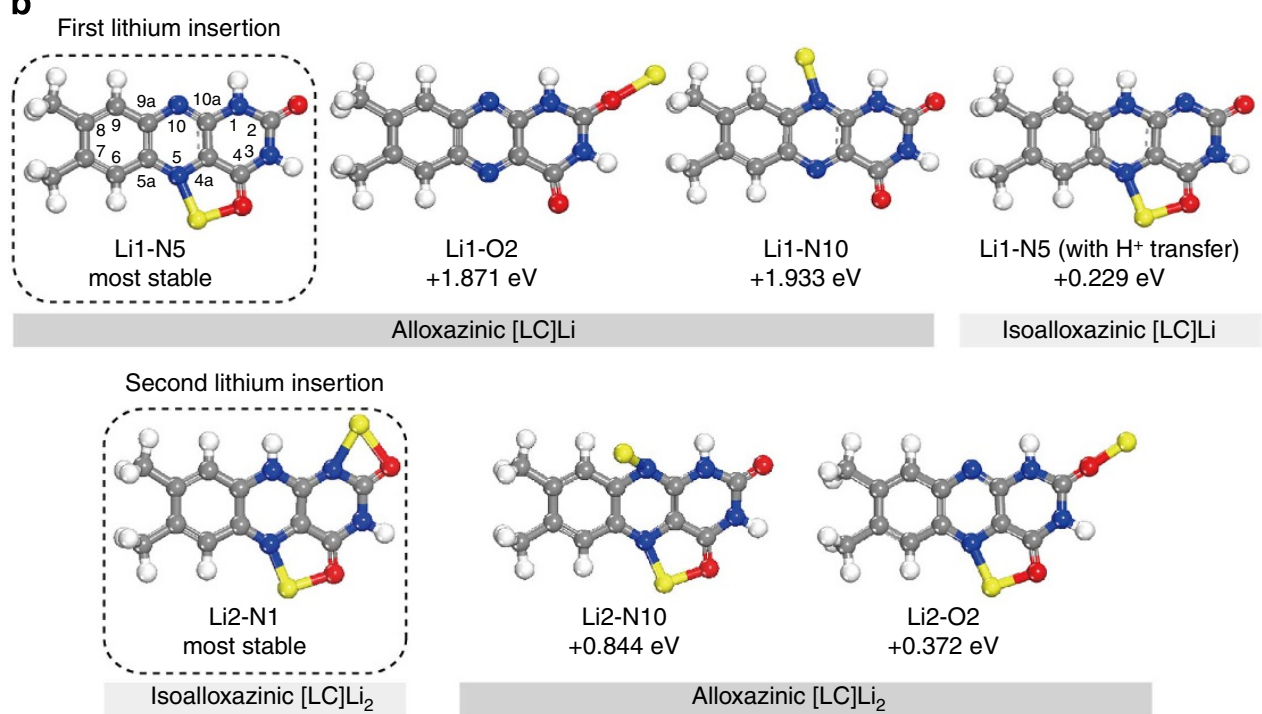

Alloxazinic $[\mathrm{LC}] \mathrm{Li}_{2}$

Figure 3 | DFT calculation on LC during redox reactions. (a) HOMO plots of LC and doubly reduced LC tautomers. (b) DFT energy comparison for various forms of $[\mathrm{LC}] \mathrm{Li}$ (upper) and $[\mathrm{LC}] \mathrm{Li}_{2}$ (lower) on two-lithium-coupled two-electron reduction.

favoured over the isoalloxazinic tautomer by $0.23 \mathrm{eV}$ under vacuum. In a more realistic simulation in the presence of electrolytes, however, we found notable distinction in preferred configuration (Supplementary Figs 4 and 5). While the alloxazinic form of [LC]Li was still preferred in a nonpolar solvent $(\varepsilon: 3.04)$ by $0.101 \mathrm{eV}$ similar to the result in vacuum condition, the isoalloxazinic form became more stable by $0.016 \mathrm{eV}$ in a polar solvent ( $\varepsilon: 78.4)$. This result implies that the choice of electrolyte strongly affects the electrochemical reaction path of tautomeric redox compounds. For the fully discharged $[\mathrm{LC}] \mathrm{Li}_{2}$, the second $\mathrm{Li}$ prefered to be located between $\mathrm{N} 1$ and $\mathrm{O} 2$ more than other positions, which accompanied proton transfer from N1 to N10. Furthermore, the charge densities successively increased on nitrogen and oxygen atoms during two-step lithiation (Supplementary Fig. 5). These results agree with the aforementioned ex situ spectroscopy results and confirm that nitrogen and oxygen atoms work as collaborative centres for the tautomerisminvolving redox reaction.

Molecular simplification to minimal diazabutadiene redox unit. Inspired by the remarkable electrochemical activity of LC, we further tested ALX and LMZ with theoretical capacities of 250 and $327 \mathrm{mAhg}^{-1}$, respectively, assuming that they store two lithium atoms per molecule similar to rivoflavin and LC electrodes. The feasiblity of the electrochemical activity was first screened by computationally reducing each molecule. In the HOMO plot of the reduced ALX and LMZ, the effective delocalization of electron clouds was observed up to two electrons, suggesting that they can be utilized as redox centres for two lithium-ion storage (Fig. 4a). DFT calculations with actual lithium occupancies in each molecule confirmed that two lithium ions could be stored in both ALX and LMZ by undergoing similar tautomerism-accompanied redox reactions as LC (Supplementary Figs 6 and 7).

We next explored the electrochemcal activity of ALX and LMZ electrodes in Li cells. As predicted by our calculations, more than one lithium ion and electron were reversibly stored in each molecule of ALX and LMZ, respectively. The ALX/Li cell showed a specific capacity of $181 \mathrm{mAhg}^{-1}$ at $20.0 \mathrm{mAg}^{-1}$ current density, corresponding to $1.45 \mathrm{Li}$ atoms per molecule (Fig. 4b, left). The GITT measurement with a lower current rate indicated that a capacity of $211 \mathrm{mAh} \mathrm{g}^{-1}(1.69 \mathrm{Li} / \mathrm{ALX})$ could be reversibly delivered. Analogous to the LC electrode, the electrochemical profile could be divided into two redox steps with average OCVs of 2.52 and $2.42 \mathrm{~V}$, respectively, which implied that two consecutive one-electron transfer reactions similarly occurred for ALX as well. In contrast, the LMZ electrode showed slightly different electrochemical profiles compared with LC and ALX with relatively larger polarizations (Fig. 4b, right). At room temperature, the LMZ/Li cell delivered a reversible capacity of $154 \mathrm{mAhg}^{-1}$, which is equivalent to only $0.94 \mathrm{Li}$ atom per molecule. The limited capability of lithium storage accompanied with the large polarization of $\sim 1.6 \mathrm{~V}$ (Supplementary Fig. 8) indicated that the relatively low capacity of the LMZ electrode may stem from the slow electrochemical reaction of LMZ. When we offered higher temperature $\left(60^{\circ} \mathrm{C}\right)$ or sufficiently low current density (intermittent current of $10.0 \mathrm{mAg}^{-1}$; Fig. $4 \mathrm{c}$, right) to LMZ/Li cells, the electrochemical activity of LMZ improved significantly. Note that the pteridine derivatives used in this study show reasonable thermal stability up to $220^{\circ} \mathrm{C}$ (Supplementary Fig. 9). The LMZ/Li cells showed specific capacities of $251 \mathrm{mAhg}^{-1}$ (1.54 Li/LMZ) and $193 \mathrm{mAhg}^{-1}$ (1.18 Li/LMZ) under the high-temperature cycling and GITT test, respectively. It implies that two lithium ions may be stored in LMZ, which is in agreement with our calculations, however, the lithiation kinetics 
a

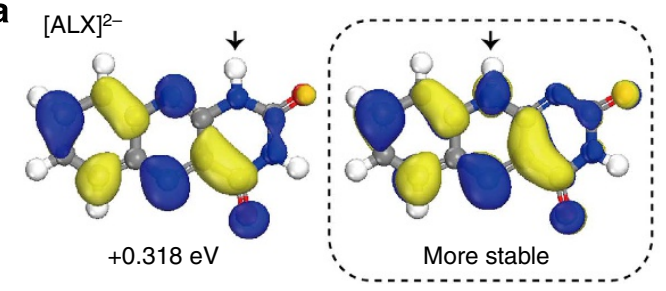

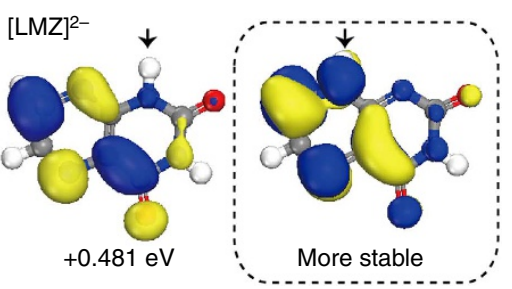

Li/lumazine

b

Li/alloxazine

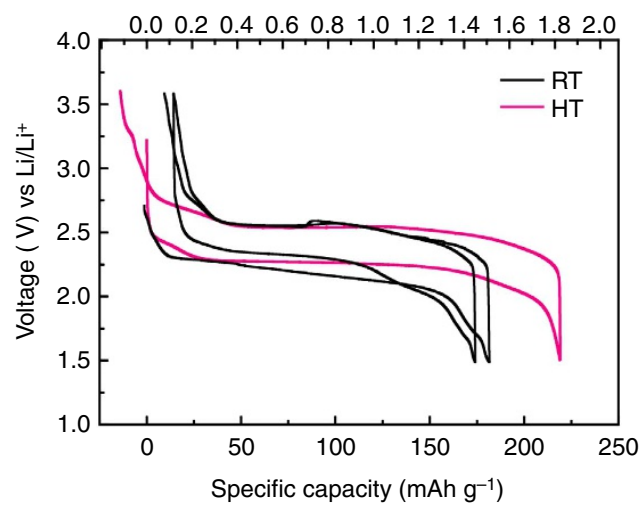

Li/alloxazine

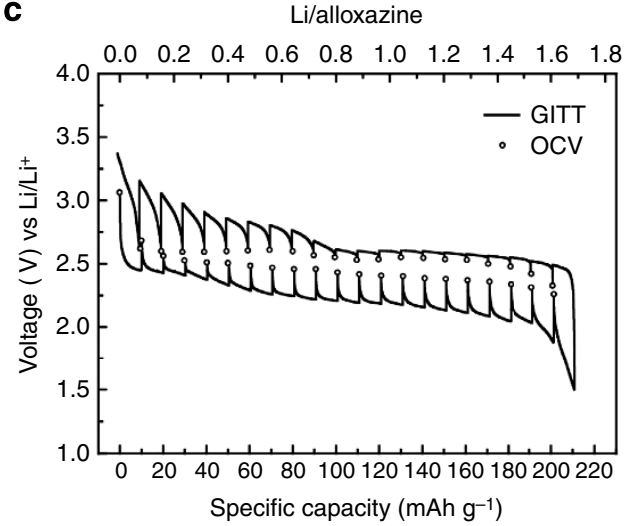

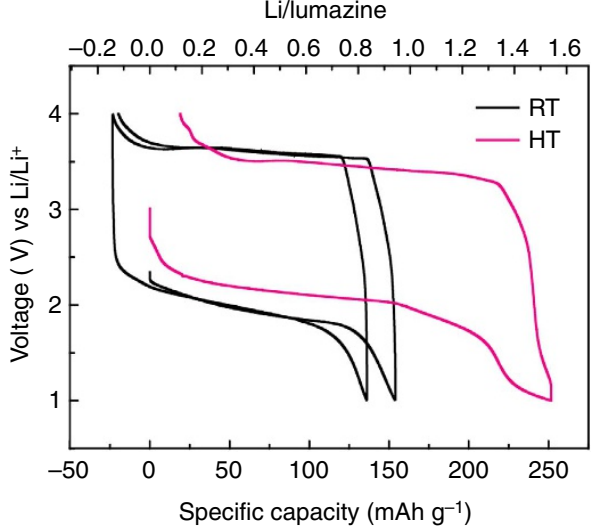

Li/lumazine

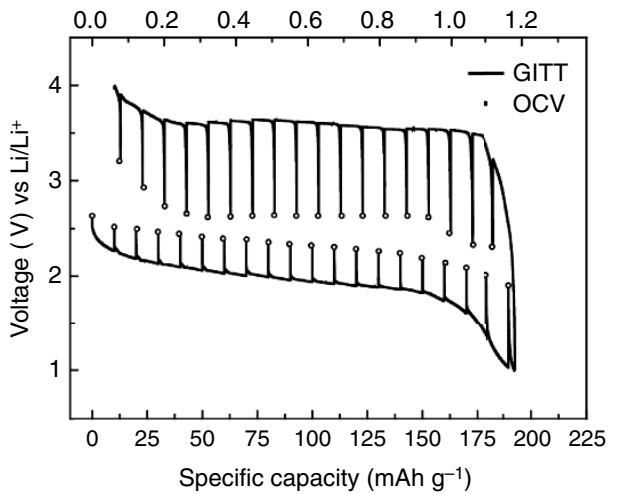

Figure 4 I Energy storage in ALX and LMZ. (a) HOMO plots for the doubly reduced species of ALX and LMZ. Black arrows indicate the proton that is bound to $\mathrm{N} 1$ or N10. (b) Capacity-voltage profiles of $\mathrm{ALX}$ and $\mathrm{LMZ}$ electrodes at room temperature (black) and $60{ }^{\circ} \mathrm{C}$ (red). (c) GITT profiles of the ALX/Li cells (left) and LMZ/Li cells (right).

were slow. The underlying reason for the slow lithiation kinetics particularly for the LMZ electrode is not fully understood yet. However, we speculate that the smaller molecular framework of LMZ compared with other molecules may induce comparatively stronger electron repulsion within the molecules when accepting two electrons. In the absence of the anellated benzo ring, the small conjugated system of LMZ is likely to show less-efficient accommodation of electrons and lithium ions at the diazabutadiene motif, thereby resulting in the large polarization. This suggests that in the molecular design of the organic electrode, the power density can be sacrificed to increase the energy density as the size of the molecule decreases. While the GITT measurement indicated that lithium insertion takes place at an average potential of 2.50 and $2.20 \mathrm{~V}$ in the LMZ electrode, the large polarization makes it less appealing for practical batteries.

Enhanced battery performance with modified electrode design. Another attractive aspect of pteridine derivative electrodes is that the electrochemical performance can be easily improved by employing a modified electrode preparation. Taking advantage of abundant electron clouds in pi orbitals of aromatic pteridines, graphitic nanocarbons can immobilize the molecules through strong $p i-p i$ interaction, which provide the redox centres with stable anchoring and a rapid electrical path. The simple solution-based process of mixing carbon nanotubes (CNTs) and organic powder followed by vacuum filtration produced highly conductive, free-standing paper, which is directly applicable as an additive-free electrode, in which the pteridine molecules were fixed onto the surface of CNTs (Supplementary Fig. 10) ${ }^{14}$. As a result of the hybridization, the redox molecules are reassembled from crystalline particles to nanolayers on the surfaces of CNTs (Supplementary Fig. 11). We found that the hybrid electrodes exhibited dramatically improved rate capability and cyclability without changes in redox potentials (Supplementary Fig. 12). The rate capabilities of the hybrid electrodes at different $\mathrm{C}$ rates are illustrated in Fig. 5a,b. At a current density of $220 \mathrm{mAh} \mathrm{g}^{-1}$, which is equivalent to a $1 \mathrm{C}$ rate, the LC hybrid electrode showed a specific capacity of $215 \mathrm{mAhg}^{-1}$. Compared with the pristine electrodes made of powdered compounds, the charge/discharge profiles of hybrid electrodes are sloppier, which is attributable to the reduced crystallinity and particle size of the active materials, as well as to the extra capacity from CNTs $\left(\sim 18 \mathrm{mAhg}^{-1}\right.$; Supplementary Fig. 13). Even at a high current density of $10.0 \mathrm{~A} \mathrm{~g}^{-1}$ corresponding to a $45.5 \mathrm{C}$ rate, LC provided a specific 

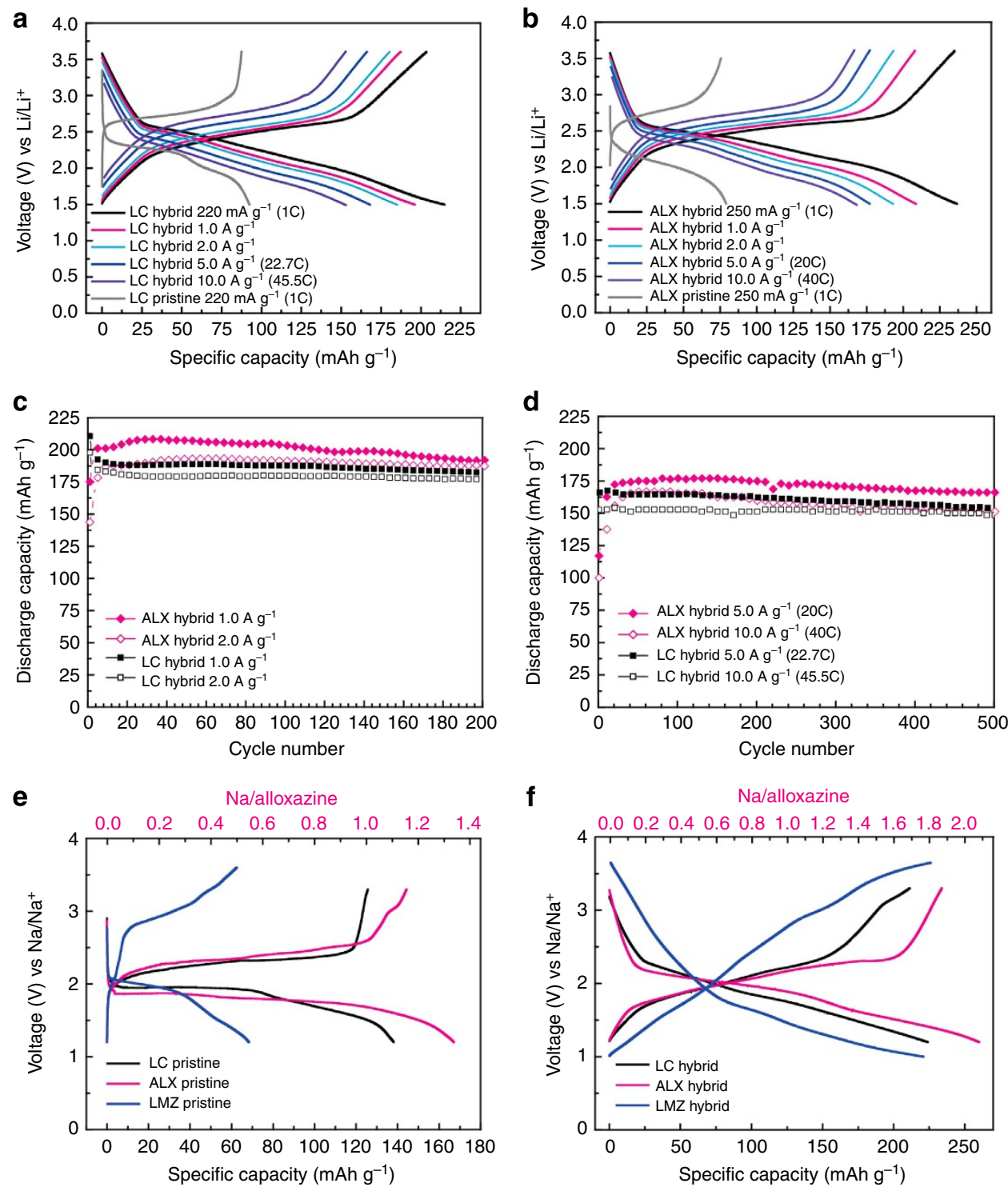

Figure 5 | Battery performances of modified pteridine electrodes. (a) Capacity-voltage profiles of LC-CNT hybrid electrodes (LC hybrid) at various current rates and a pristine LC electrode at a 1 C rate. (b) Capacity-voltage profiles of ALX-CNT hybrid electrodes (ALX hybrid) at various current rates and a pristine ALX electrode at a $1 \mathrm{C}$ rate. Capacity retention of the LC hybrid and ALX hybrid (c) at 1.0 and $2.0 \mathrm{Ag}^{-1}$ for 200 cycles and (d) at 5.0 and $10.0 \mathrm{Ag}^{-1}$ for 500 cycles. Capacity-voltage profiles of (e) pristine pteridine derivatives at $20 \mathrm{mAg}^{-1}$ and (f) pteridine hybrid electrodes at $50 \mathrm{mAg}-1$ in sodium rechargeable batteries.

capacity of $153 \mathrm{mAh} \mathrm{g}^{-1}$, corresponding to $71.1 \%$ of the capacity at the $1 \mathrm{C}$ rate (Fig. 5a). The ALX hybrid electrode could deliver a specific capacity of $236 \mathrm{mAhg}^{-1}$ at the $1 \mathrm{C}$ rate $\left(250 \mathrm{mAg}^{-1}\right)$ and $168 \mathrm{mAh} \mathrm{g}^{-1}$ at the $10 \mathrm{C}$ rate, as shown in Fig. 5b. Moreover, the LC and ALX hybrid electrodes showed remarkable capacity retentions compared with the pristine electrodes (Fig. 5c; Supplementary Fig. 14). After 200 cycles at 1.0 and $2.0 \mathrm{Ag}^{-1}$, the LC hybrids showed 93 and $96 \%$ capacity retention, and the ALX hybrids showed 92 and $97 \%$ capacity retention, respectively. Even after cycling up to 500 times, the LC hybrid electrodes exhibited the capacity retention above 93 and $96 \%$ at a high rate of 5.0 and $10.0 \mathrm{Ag}^{-1}$ (Fig. 5d), respectively. In addition, the ALX hybrid electrodes retained 93 and $91 \%$ of their initial capacity at 5.0 and $10.0 \mathrm{Ag}^{-1}$, respectively. To the best of our knowledge, the capacity retention of $96 \%$ up to 500 cycles at $10.0 \mathrm{Ag}^{-1}$ is the best and the longest cycling performance among the organic molecule-based cathode materials reported thus far ${ }^{11,15,16,36}$.
Sodium storage in pteridine systems. As the limited abundance of lithium increases the need for alternative carrier ions in rechargeable battery systems $\mathrm{s}^{37}$, we report that pteridine-based organic electrodes are also electrochemically active to sodium. As potential cathodes in sodium rechargeable batteries, we evaluated the electrochemical reactivity of pteridine derivatives to sodium using a half cell with sodium metal. Figure $5 \mathrm{e}$ shows that pteridine derivatives are capable of reversibly taking up and releasing two sodium ions, representing them as versatile electrodes in rechargeable batteries. In sodium batteries, LC and ALX exhibited a specific capacity of 138 and $168 \mathrm{mAhg}^{-1}$, respectively, and LMZ delivered a slightly lower capacity of $70 \mathrm{mAhg}^{-1}$ at a current density of $10.0 \mathrm{mAg}^{-1}$. By incorporating CNTs during electrode preparation, the deliverable capacities were significantly improved to 222,255 and $220 \mathrm{mAh} \mathrm{g}^{-1}$ even at $50.0 \mathrm{~mA} \mathrm{~g}^{-1}$ for LC, ALX and LMZ, respectively (Fig. $5 \mathrm{f}$ ), although the capacity could not be stably retained during repeated cycling $(\sim 50 \%$ retention after 20 cycles 
for all compounds). The limited cyclability could be attributable to the non-optimized $\mathrm{Na}$ electrolytes of the cell and the large strain of the electrode materials during the insertion of large sodium ions, which may induce the isolation of the active materials from the electrodes. While further studies are required, the sodium-organic battery system is promising for sustainable energy storage systems, since it is based on abundant sodium elements on earth and does not contain transition metals ${ }^{38-40}$. The utilization of diazabutadiene redox cycling should provide potential opportunities for sodium-organic battery systems.

\section{Discussion}

We described a molecular simplification strategy using tailored pteridine derivatives that contain the redox-active diazabutadiene motif of flavins to develop green and high-performance energy storage systems. For the first time, reversible tautomerism between alloxazinic and isoalloxazinic structures in pteridine derivatives during lithium-coupled electron uptake and donation was demonstrated through analyses of both ex situ characterization and DFT calculations. The pteridine-based electrode with conductive CNT scaffolds exhibited high gravimetric energy density up to $533 \mathrm{Wh} \mathrm{kg}^{-1}$ within a 1-h discharge, and the gravimetric energy density of $348 \mathrm{Wh} \mathrm{kg}^{-1}$ was demonstrated even at the high powers greater than $20 \mathrm{~kW} \mathrm{~kg}^{-1}$. Up to $96 \%$ capacity was retained in the pteridine-based hybrid electrode after cycling 500 times at $10.0 \mathrm{Ag}^{-1}$, which is the best and longest cycling performance at such a high rate among organic moleculebased cathode materials. Also, the utilization of diazabutadiene redox cycling in sodium-ion batteries was first proposed, while further study is required to overcome the capacity fading. The current study suggests that various unexplored redox molecules from natural energy transduction systems can provide unlimited opportunities to design sustainable electrodes with superior performances beyond the conventional electrode materials of rechargeable lithium/sodium-ion batteries.

\begin{abstract}
Methods
Electrochemistry. Cyclic voltammograms of pteridine derivatives $(0.5 \mathrm{mM})$ were captured using TBAP and $\mathrm{LiClO}_{4}$ electrolytes $(0.1 \mathrm{M})$ in DMF solution under $\mathrm{N}_{2}$ atmosphere. A three-electrode system ( $\mathrm{Pt}$ counter electrode, $\mathrm{Ag} / \mathrm{AgCl}$ reference electrode, glassy carbon working electrode) was employed and the scan rate was $100 \mathrm{mV} \mathrm{s}^{-1}$. Electrochemical profiles of LC, ALX and LMZ powder samples were measured versus a Li metal foil (Hohsen, Japan) and a Na metal cube (Sigma Aldrich, USA) in coin-type cells (CR2016). The electrodes were fabricated by mixing $40 \% \mathrm{w} / \mathrm{w}$ active materials, $40 \% \mathrm{w} / \mathrm{w}$ conductive carbon (Super P) and $20 \%$ $\mathrm{w} / \mathrm{w}$ polytetrafluoroethylene (PTFE) binder for both $\mathrm{Li}$ - and $\mathrm{Na}$-ion cells. We adjusted the total amounts of electrodes within the range of $6.0 \pm 1.0 \mathrm{mg}$ per cell. The electrodes were dried overnight at $60^{\circ} \mathrm{C}$ under vacuum. In the modified electrode preparation, hybrid electrodes were produced by mixing the organic compounds and single-walled CNTs in acetone using a sonicator followed by vacuum filtration. The resulting self-standing paper was directly used as an electrode without current collectors and additives for both $\mathrm{Li}$ - and $\mathrm{Na}$-ion cells. According to the elemental analysis, the portions of active materials in the hybrid electrodes were 43,41 and $35 \mathrm{wt} \%$ for LC, ALX and LMZ, respectively. The average weight of the free-standing electrodes was $\sim 1.0 \mathrm{mg}$ per cell. A porous glass microfiber membrane (GF/F; Whatman, UK) was used as a separator in both the $\mathrm{Li}$ - and Na-ion cells. The electrolytes were $1 \mathrm{M} \mathrm{LiPF}_{6}$ in tetraethylene glycol dimethyl ether (TEGDME, Techno Semichem, Korea) for Li-ion cells and $1 \mathrm{M}$ $\mathrm{NaPF}_{6}$ in diethylene glycol dimethyl ether (DEGDME) for Na-ion cells. The cells were assembled under inert atmosphere in an Ar-filled glove box. Galvanostatic measurements were performed at various constant current densities on a battery test system (Won-A, Korea).
\end{abstract}

Ex situ electrode characterization. For ex situ analyses, the electrodes were prepared by disassembling coin cells at different states of charge (as-prepared, half-discharged and fully discharged to $1.6 \mathrm{~V}$, half-charged and fully recharged to $3.6 \mathrm{~V}$ ) followed by rinsing the electrodes with dimethyl carbonate. To prevent exposure to air, all samples were sealed in an Ar-filled glove box. XPS measurements were conducted with a Thermo VG Scientific Sigma Probe spectrometer (UK) equipped with a microfocus monochromated X-ray source $(90 \mathrm{~W})$. Binding energies were referenced to the $\mathrm{C}-\mathrm{C}$ bond of the $\mathrm{C} 1 \mathrm{~s}$ region at $284.5 \mathrm{eV}$. Fourier transform infrared spectra were recorded with pellets made of active powders scraped out of the electrodes and $\mathrm{KBr}$ powder on an FT/IR-4200 (Jasco, Japan) at a resolution of $2 \mathrm{~cm}^{-1}$ under vacuum. For fluorescence spectroscopy, samples were immersed in degassed deionized water under a nitrogen atmosphere, resulting in solubilized active molecules. Fluorescence spectra were obtained using a V/650 spectrophotometer (Jasco) in the range of $400-600 \mathrm{~nm}$.

Computational details. Geometry optimization and energy calculations on the molecules were conducted with spin-unrestricted DFT using the Gaussian 09 quantum chemistry package ${ }^{41}$ based on the Becke-Lee-Yang-Parr (B3LYP) hybrid exchange-correlation functional ${ }^{42-44}$ and the standard TZVP basis set ${ }^{45-47}$. The site and sequence of lithium occupation during the redox reaction were determined by comparing DFT energies of possible reduced forms of molecules bonded to one or two lithium ions. Atomic charge was calculated using Mulliken population analysis ${ }^{48}$. A detailed procedure and the results of DFT calculations are discussed in the Supplementary Information. The HOMO plots were generated with the cubegen utility, which was embedded in Gaussian 09 (ref. 41) and visualized with the Materials Studio program.

\section{References}

1. Liang, Y., Tao, Z. \& Chen, J. Organic electrode materials for rechargeable lithium batteries. Adv. Energy Mater. 2, 742-769 (2012).

2. Reddy, A. L. M. et al. Lithium storage mechanisms in purpurin based organic lithium ion battery electrodes. Sci. Rep. 2, 960 (2012).

3. Song, Z. \& Zhou, H. Towards sustainable and versatile energy storage devices: an overview of organic electrode materials. Energy Environ. Sci. 6, 2280-2301 (2013).

4. Poizot, P. \& Dolhem, F. Clean energy new deal for a sustainable world: from non- $\mathrm{CO}_{2}$ generating energy sources to greener electrochemical storage devices. Energy Environ. Sci. 4, 2003-2019 (2011).

5. Chen, $\mathrm{H}$. et al. From biomass to a renewable $\mathrm{Li}_{\mathrm{x}} \mathrm{C}_{6} \mathrm{O}_{6}$ organic electrode for sustainable Li-ion batteries. ChemSusChem 1, 348-355 (2008).

6. Milczarek, G. \& Inganas, O. Renewable cathode materials from biopolymer/ conjugated polymer interpenetrating networks. Science 335, 1468-1471 (2012).

7. Han, X., Chang, C., Yuan, L., Sun, T. \& Sun, J. Aromatic carbonyl derivative polymers as high-performance Li-ion storage materials. Adv. Mater. 19, 1616-1621 (2007).

8. Song, Z., Zhan, H. \& Zhou, Y. Polyimides: promising energy-storage materials. Angew. Chem. Int. Ed. 49, 8444-8448 (2010).

9. Huskinson, B. et al. A metal-free organic-inorganic aqueous flow battery. Nature 505, 195-198 (2014).

10. Wang, S. et al. Organic $\mathrm{Li}_{4} \mathrm{C}_{8} \mathrm{H}_{2} \mathrm{O}_{6}$ nanosheets for lithium-ion batteries. Nano Lett. 13, 4404-4409 (2013).

11. Huang, W. et al. Quasi-solid-state rechargeable lithium-ion batteries with a calix[4]quinone cathode and gel polymer electrolyte. Angew. Chem. 125, 9332-9336 (2013).

12. Nishida, S., Yamamoto, Y., Takui, T. \& Morita, Y. Organic rechargeable batteries with tailored voltage and cycle performance. ChemSusChem 6, 794-797 (2013).

13. Morita, Y. et al. Organic tailored batteries materials using stable open-shell molecules with degenerate frontier orbitals. Nat. Mater. 10, 947-951 (2011).

14. Lee, M. et al. Organic nanohybrids for fast and sustainable energy storage. $A d v$ Mater. 26, 2558-2565 (2014)

15. Wu, H. et al. Flexible and binder-free organic cathode for high-performance lithium-ion batteries. Adv. Mater. 26, 3338-3343 (2014).

16. Nokami, T. et al. Polymer-bound pyrene-4,5,9,10-tetraone for fast-charge and -discharge lithium-ion batteries with high capacity. J. Am. Chem. Soc. 134, 19694-19700 (2012).

17. Luo, C. et al. Self-assembled organic nanowires for high power density lithium ion batteries. Nano Lett. 14, 1596-1602 (2014).

18. Kaim, W., Schwederski, B., Heilmann, O. \& Hornung, F. M. Coordination compounds of pteridine, alloxazine and flavin ligands: structures and properties. Coord. Chem. Rev. 182, 323-342 (1999).

19. Sakai, K., Nagahara, K., Yoshii, Y., Hoshino, N. \& Akutagawa, T. Structural and spectroscopic study of 6,7-dicyano-substituted lumazine with high electron affinity and proton acidity. J. Phys. Chem. A 117, 3614-3624 (2013).

20. Miura, R. Versatility and specificity in flavoenzymes: control mechanisms of flavin reactivity. Chem. Rec. 1, 183-194 (2001).

21. Joosten, V. \& van Berkel, W. J. H. Flavoenzymes. Curr. Opin. Chem. Biol. 11, 195-202 (2007).

22. Tan, S. L. J. \& Webster, R. D. Electrochemically induced chemically reversible proton-coupled electron transfer reactions of riboflavin (vitamin B2). J. Am. Chem. Soc. 134, 5954-5964 (2012).

23. Lee, M. et al. Redox cofactor from biological energy transduction as molecularly tunable energy-storage compound. Angew. Chem. Int. Ed. 52, 8322-8328 (2013).

24. Sikorska, E. et al. Hydrogen-bonded complexes of lumichrome. J. Phys. Chem. A 109, 1785-1794 (2005) 
25. Moyon, N. S. \& Mitra, S. Fluorescence solvatochromism in lumichrome and excited-state tautomerization: a combined experimental and DFT study. J. Phys. Chem. A 115, 2456-2464 (2011).

26. Sikorska, E. et al. Ground- and excited-state double proton transfer in lumichrome/acetic acid system: theoretical and experimental approach. J. Phys. Chem. A 109, 11707-11714 (2005).

27. Vieira Ferreira, L. F. et al. Surface photochemistry: alloxazine within nanochannels of $\mathrm{Na}^{+}$and $\mathrm{H}^{+}$ZSM-5 zeolites. Phys. Chem. Chem. Phys. 11, 5762-5772 (2009)

28. Hernández-Burgos, K., Rodríguez-Calero, G. G., Zhou, W., Burkhardt, S. E. \& Abruña, H. D. Increasing the gravimetric energy density of organic based secondary battery cathodes using small radius cations $\left(\mathrm{Li}^{+}\right.$and $\left.\mathrm{Mg}^{2}+\right) . J . A m$. Chem. Soc. 135, 14532-14535 (2013).

29. Castillo-Martínez, E., Carretero-González, J. \& Armand, M. Polymeric Schiff bases as low-voltage redox centers for sodium-ion batteries. Angew. Chem. Int. Ed. 53, 5341-5345 (2014).

30. Armand, M. et al. Conjugated dicarboxylate anodes for Li-ion batteries. Nat. Mater. 8, 120-125 (2009).

31. Bhargava, G., Ramanarayanan, T. A. \& Bernasek, S. L. Imidazole - Fe interaction in an aqueous chloride medium: effect of cathodic reduction of the native oxide. Langmuir 26, 215-219 (2009).

32. Lee, T. H., Colton, R. J., White, M. G. \& Rabalais, J. W. Electronic structure of hydrazoic acid and the azide ion from X-ray and ultraviolet electron spectroscopy. J. Am. Chem. Soc. 97, 4845-4851 (1975).

33. Takahashi, M., Ishikawa, Y., Nishizawa, J.-i. \& Ito, H. Low-frequency vibrational modes of riboflavin and related compounds. Chem. Phys. Lett. 401, 475-482 (2005).

34. Wolf, M. M. N., Schumann, C., Gross, R., Domratcheva, T. \& Diller, R. Ultrafast infrared spectroscopy of riboflavin: dynamics, electronic structure, and vibrational mode analysis. J. Phys. Chem. B 112, 13424-13432 (2008).

35. Liang, Y., Zhang, P. \& Chen, J. Function-oriented design of conjugated carbonyl compound electrodes for high energy lithium batteries. Chem. Sci. 4, 1330-1337 (2013).

36. Song, Z. et al. Polymer-graphene nanocomposites as ultrafast-charge and -discharge cathodes for rechargeable lithium batteries. Nano Lett. 12, 2205-2211 (2012).

37. Kim, S.-W., Seo, D.-H., Ma, X., Ceder, G. \& Kang, K. Electrode materials for rechargeable sodium-ion batteries: potential alternatives to current lithium-ion batteries. Adv. Energy Mater. 2, 710-721 (2012).

38. Yao, M. et al. Indigo carmine: an organic crystal as a positive-electrode material for rechargeable sodium batteries. Sci. Rep. 4, 3650 (2014).

39. Sakaushi, K. et al. Aromatic porous-honeycomb electrodes for a sodiumorganic energy storage device. Nat. Commun. 4, 1485 (2013).

40. Wang, S. et al. All organic sodium-ion batteries with $\mathrm{Na}_{4} \mathrm{C}_{8} \mathrm{H}_{2} \mathrm{O}_{6}$. Angew. Chem. 126, 6002-6006 (2014).

41. Frisch, M. et al. Gaussian 09 revision D. 01 (Gaussian Inc., 2009).
42. Lee, C., Yang, W. \& Parr, R. Development of the Colle-Salvetti correlationenergy formula into a functional of the electron density. Phys. Rev. B 37, 785-789 (1988).

43. Stephens, P. J., Devlin, F. J., Chabalowski, C. F. \& Frisch, M. J. Ab initio calculation of vibrational absorption and circular dichroism spectra using density functional force fields. J. Phys. Chem. 98, 11623-11627 (1994).

44. Becke, A. D. Density-functional thermochemistry. III. The role of exact exchange. J. Chem. Phys. 98, 5648-5652 (1993).

45. Schäfer, A., Huber, C. \& Ahlrichs, R. Fully optimized contracted Gaussian basis sets of triple zeta valence quality for atoms Li to Kr. J. Chem. Phys. 100, 5829-5835 (1994).

46. Schäfer, A., Horn, H. \& Ahlrichs, R. Fully optimized contracted Gaussian basis sets for atoms Li to Kr. J. Chem. Phys. 97, 2571-2577 (1992).

47. Klaumúnzer, B., Króner, D. \& Saalfrank, P. (TD-) DFT calculation of vibrational and vibronic spectra of riboflavin in solution. J. Phys. Chem. B 114, 10826-10834 (2010).

48. Mulliken, R. S. Electronic population analysis on LCAO-MO molecular wave functions. I. J. Chem. Phys. 23, 1833-1840 (1955).

\section{Acknowledgements}

This study was supported by development of organic electrode materials for lithium ion batteries project (UE1240220D) funded by the Agency for Defense Development (ADD), Republic of Korea. This work was also supported by the Intelligent Synthetic Biology Center of Global Frontier R\&D Project (2011-0031957), and the Human Resources Development program (20124010203320) of the Korea Institute of Energy Technology Evaluation and Planning (KETEP) grant funded by the Korea government Ministry of Trade, Industry and Energy, Republic of Korea.

\section{Author contributions}

J.H. and M.L. conceived the research. K.K. and C.B.P. supervised the research. M.L. and J.H. designed and performed the experiments and analysed the data. B.L. and D.-H.S. conducted DFT calculations. J.H., M.L., C.B.P. and K.K. wrote the manuscript. All authors discussed the results and commented on the manuscript.

\section{Additional information}

Supplementary Information accompanies this paper at http://www.nature.com/ naturecommunications

Competing financial interests: The authors declare no competing financial interests.

Reprints and permission information is available online at http://npg.nature.com/ reprintsandpermissions/

How to cite this article: Hong, J. et al. Biologically inspired pteridine redox centres for rechargeable batteries. Nat. Commun. 5:5335 doi: 10.1038/ncomms6335 (2014). 